\title{
Advances in fetal surgery
}

\author{
Kathryn M. Maselli ${ }^{1}$, Andrea Badillo \\ ${ }^{1}$ Department of Surgery, Medstar Georgetown University Hospital, Washington DC 20007, USA; ${ }^{2}$ Division of Pediatric Surgery, Children's National \\ Medical Center, Washington DC 20010, USA \\ Contributions: (I) Conception and design: All authors; (II) Administrative support: None; (III) Provision of study materials or patients: None; (IV) \\ Collection and assembly of data: None; (V) Data analysis and interpretation: None; (VI) Manuscript writing: All authors; (VII) Final approval of \\ manuscript: All authors. \\ Correspondence to: Andrea Badillo, MD. Division of Pediatric Surgery, Children's National Medical Center, 111 Michigan Avenue NW, Suite W4-200, \\ Washington DC 20010, USA. Email: abadillo@childrensnational.org.
}

\begin{abstract}
Historically, the gold standard for the treatment of congenital malformations has been planned delivery at tertiary care center with attempted post-natal repair or amelioration of the lesion. Over the last few decades however, rapid advances in imaging and instrumentation technology combined with superior knowledge of fetal pathophysiology has led to the development of novel intrauterine interventions for most common fetal anomalies. Great success has already been seen the treatment of previous devastating anomalies such as myelomeningocele (MMC), congenital cystic malformations of the lung, twin-twin transfusion, and sacrococcygeal teratomas. Although still limited, these innovative techniques have unique potential to improve outcomes in the most devastating fetal anomalies.
\end{abstract}

Keywords: Congenital lung malformation (CLM); nervous system malformations; fetal therapy; fetoscopic surgery; fetal ultrasonography

Submitted Sep 27, 2016. Accepted for publication Oct 19, 2016.

doi: $10.21037 / \mathrm{atm} .2016 .10 .34$

View this article at: http://dx.doi.org/10.21037/atm.2016.10.34

Until approximately 40 years ago, the developing human fetus remained enigmatic and poorly studied, shielded from observation and therapy by the uterus. The development of prenatal ultrasound (US) in the 1970s brought forth rapid advances in fetal imaging and sampling techniques, allowing for better understanding of fetal pathophysiology. Despite advances in post-natal therapies, congenital birth defects remain a major cause of morbidity and mortality, affecting approximately $3 \%$ of infants in the US alone (1). Substantial advances in imaging modalities, instrumentation, and surgical technique have led remarkable interventions of the unborn fetus. Currently, there are multiple fetal anomalies, which may be treated with selective antenatal surgical therapy. While the standard of treatment for most fetal anomalies remains planned delivery at a tertiary medical center with appropriate neonatal therapy, a growing subset of anatomical anomalies may benefit from surgical intervention before birth.

\section{Imaging modalities}

Advances in fetal therapy would have been impossible without the preceding advancements in imaging that have taken place over the last 40 years. The development of prenatal US has propelled advances in prenatal diagnostics such as amniocentesis. The use of prenatal US also allows for better understanding of the fetal pathophysiology with respect to common fetal anomalies. This critical knowledge paved the way for developing therapies aimed for intervention during gestation rather than postnatally.

More than $80 \%$ of the most common fetal anomalies develop before the twelfth week of gestation. Unfortunately, this is when visualization of the developing fetus is most difficult (2). Nevertheless, US offers several advantages for fetal evaluation including low cost, widespread availability, multiplanar capability, and real time image display. As ultrasound technology has improved the sensitivity of US for diagnosing the common fetal malformations has 
improved. A large scale prospective study of 1,148 singleton gestations found the sensitivity of $2 \mathrm{D}$ ultrasound to be $50 \%$ at 11-14 weeks (3). Sensitivity increased to $92.8 \%$ with the addition of a second scan at 22-24 weeks (3).

Ultimately ultrasound technology has dramatically improved in quality and pixilation alongside dynamic evaluation of flow and reconstruction of multi-dimensional structures yielding an enhanced sensitivity for diagnosing common fetal malformations. The development of transvaginal US, high frequency transducers, and improved processing speed has greatly improved the ability of US to demonstrate structural abnormalities. The development of 3D and 4D ultrasound technology has further expanded the possible applications for prenatal ultrasound. Three dimensional ultrasound is now widely available and is commonly applied in the diagnosis of craniofacial, neural tube, and skeletal abnormalities (4). In particular, 3D ultrasound allows for volumetric measurements of fetal organs, such as lung volume to determine the degree of pulmonary hypoplasia in a fetus with a congenital lung malformation (CLM). Three dimensional and 4D US also has considerable applications in the diagnosis, as well as potential treatment, of congenital heart defects. Spatio-temporal image correlation allows for real-time $3 \mathrm{D}$ reconstruction of the fetal cardiac cycle from multiple planes (5). This reconstructed cycle can be used to isolate images of the five classic planes of fetal echocardiography at any point in the cycle (5). The addition of Doppler flow provides further information about cardiac function including ejection fraction and valvular regurgitation. With 4D US, the complex dynamics of small anatomic structures can be assessed in greater detail (6). The increased structural detail available through 3D/4D US is critical for surgical planning.

In addition to identifying structural abnormalities, ultrasound can be used to identify markers of fetal distress and indicators of imminent fetal demise. This allows for stratification of fetal distress to determine if and when intrauterine intervention is necessary. Doppler velocimetry, although not useful for identifying congenital heart defects, is helpful for the evaluation of fetal myocardial function (7). Venous velocimetry can be used to detect hydrops, an indicator of imminent demise due to high-output cardiac failure seen in arterio-venous shunts due to sacrococcygeal teratomas and other malformations (7).

The real-time spatiotemporal resolution of ultrasound makes it uniquely useful to guide surgical intervention. Ultrasonography is the ideal imaging modality for use in routine fetal procedures, such as amniocentesis, or more complex procedures. Ultrasound is used intraoperatively to map the placental location and select the hysterotomy site during ex utero intrapartum treatment (EXIT) procedures as well as myelomeningocele (MMC) repair and other open fetal surgeries $(8,9)$. Intraoperative US is also used to guide needle and instrument placement in fetoscopy and other procedures such as laser ablation for twin-twin transfusion (4). Four dimensional US is especially useful in procedural guidance as it provides real-time images and multiplanar views as opposed to $2 \mathrm{D}$ US. Although improvement in resolution is still needed to improve the technique, 4D US has been used to guide the cauterization of an umbilical cord in twin-twin transfusion syndrome $(6,10)$.

Another promising imaging modality for evaluation of fetal malformations is magnetic resonance imaging (MRI). Although the frequent and rapid movements of the developing fetus make classic MRI a poor imaging modality, the development of ultra-fast MRI has led to significant advances in fetal imaging. Ultrafast MRI technology acquires an MRI sequence in 20 seconds, eliminating the need for fetal immobilization during MRI (11). Although US is highly useful for evaluation of the developing fetus, there are certain limitations to the technique. In particular, beam attenuation by maternal adipose tissue, poor image quality in oligohydramnios, and obscuration of the fetal head by the maternal pelvic bones limit the scope of US (12). As such, US is not always sufficient for a definitive diagnosis and in practice fetal MRI is often used in conjunction with US to ensure accuracy of the prenatal diagnosis of congenital malformations. The advantages of MRI include better soft-tissue contrast, precise volumetric measurements, a larger field of view, and better imaging of intracranial structures (12). Coakley et al. describe the fetal MRI as most likely to impact management in patients referred for inconclusive sonographic findings (13). Fetal MRI is particularly useful in evaluation of fetuses with space occupying intrathoracic lesions due to precise measurement of fetal lung volume and the extent of pulmonary hypoplasia. It is used to calculate the relative volumes of normal and abnormal lung, conferring a prognostic value. Zamora et al found that an MRI-derived lung mass volume ration (LVMR) of $>2.0$ predicted a worse perinatal outcome with a sensitivity of $83 \%$ and specificity of $99 \%$ (14). Although US can also be used to determine lung volumes in fetuses with CLMs and congenital diaphragmatic hernias $(\mathrm{CDH})$, US is less reliable than MRI and more operator dependent (11). Advances in imaging technology and the 
approach of combining US with fetal MRI in prenatal evaluations has led to improved accuracy and precision in the early diagnosis of congenital malformations.

Improvements in image processing and reconstructive software also have important implications for the development of fetal therapies. MRI can be used to create patient specific virtual 3D modules to be used in surgical planning and intraoperative guidance. Although no data yet exists to speak to impact on outcome, there are multiple published case studies reporting the use of computer generated $3 \mathrm{D}$ models in surgical planning for fetal intervention (4). Werner et al. used $3 \mathrm{D}$ reconstructions of four fetuses with complex cervical malformations to create virtual bronchoscopies which demonstrated patent airways, allowing for planned cesarean delivery and post-natal therapy rather than EXIT procedure with good outcomes in all cases (15). Computer based 3D reconstructive models have enormous potential as fetal imaging continues to advance.

\section{EXIT procedure}

The EXIT procedure is a controlled method of delivery which utilizes deep anesthesia and uterine relaxation to maintain placental circulation preserving oxygen delivery to the neonate while complex interventions are preformed to secure the infant's airway just prior to delivery thereby minimizing the risk of hypoxemia. The EXIT procedure was initially developed as a delivery technique to reverse temporary fetal tracheal occlusion for severe CDH (16). The EXIT procedure is now most commonly used to deliver fetuses with prenatal evidence of severe airway obstruction or high likelihood of cardiopulmonary insufficiency at birth (17). Such indications include large cervical masses, CLMs, congenital high airway obstruction (CHAOS), unilateral pulmonary agenesis, and EXIT to extracorporeal membrane oxygenation (ECMO) in fetuses with CDH (17). The EXIT procedure requires a coordinated, multidisciplinary approach with anesthesiologists, pediatric surgeons, obstetricians, experienced ultrasonographers, and neonatologists all present during the procedure. Anesthesia considerations are critical to minimize uterine tone without compromising maternal blood pressure and consequently placental perfusion of the fetus.

At the start of the procedure the mother is positioned in a slight lateral decubitus position and an extended Pfannenstiel incision is used to expose the uterus (18). Ultrasound is used to map placental borders and select the optimal hysterotomy site. The hysterotomy is created using a uterine stapler with absorbable sutures that fixes the amniotic membranes to the uterine wall, providing both access to the uterine cavity and hemostasis (19). Once the fetus is exposed, fetal anesthesia is administered and fetal monitors placed. The fetal head is delivered and direct laryngoscopy can be performed with additional interventions as necessary (18). Once the airway is secured, further surgical intervention with appropriate fetal exposure can be carried out such as lung resection in fetuses with CLM. Upon completion of the procedure, the umbilical cord is cut and the infant delivered with appropriate postdelivery management by the neonatologist (18). The EXIT procedure represents an excellent technique for management of neonates with potentially life-threatening airway compromise. At the University of California, San Francisco (UCSF), between 1993-2003, 52 EXIT procedures were performed with 51 patients born alive and $52 \%$ alive at long term follow up (18). At Children's Hospital of Philadelphia (CHOP), 31 EXIT procedures were performed between 1996-2002 with only one fetal death (17).

Although safe delivery of the fetus is the desired goal, maternal safety is the highest priority throughout the procedure. A study of 34 women who underwent EXIT procedure found an increased risk of post-operative wound complications but no difference in length of hospital stay (20). There were no maternal deaths in either study $(17,18)$. A prospective study of women who underwent EXIT procedure had no issues with future fertility and had similar complications rates in subsequent pregnancies to the general population (21).

\section{Open fetal surgery}

Open fetal surgery allows for surgical intervention on the fetus while maintaining placental perfusion with the goal of continuing intrauterine gestation as long as possible post-operatively. The technical aspects of the procedure are similar to those involved in the EXIT procedure. The uterus is exposed via a low transverse incision and the same specialized uterine staplers are employed to fix the membranes to the uterine opening, thereby preventing membrane separation, and to create a hemostatic hysterotomy (22). The fetus is appropriately positioned for the desired intervention and fetal monitors placed. The uterus is continually infused with warmed lactated Ringers solution to maintain the uterine volume and prevent uterine 
contraction (22). On completion of the procedure, the uterus is closed and warmed LR with antibiotics is infused into the uterus to replace lost amniotic fluid (22). Postoperatively both mother and fetus require close monitoring and a strict tocolytic regimen. The fetus is usually delivered by planned cesarean section at 36 weeks of gestation. Earlier delivery may be required if pregnancy-associated complications develop. The post-operative course may be complicated by amniotic fluid leaks, chorioamniotic separation, and development of non-cardiogenic pulmonary edema in the mother, thought to be due to release of vasoactive agents from disruption of the myometrium $(23,24)$. Preterm labor is the most common complication of open fetal surgery, with an average gestational age at delivery of 34 weeks and mean duration of 10 weeks from surgery to delivery in one series (22).

\section{Fetoscopic surgery}

As laparoscopic and video-assisted surgery has become more prevalent, fetoscopic surgery has been proposed as an alternative to open fetal surgery. Fetoscopic surgery avoids the maternal morbidity associated with a large laparotomy incision with the hope that avoidance of a large hysterotomy may also reduce the incidence of pre-term labor post-operatively. Similar to other fetal interventions, rigorous intraoperative monitoring of both mother and fetus is paramount and adequate tocolysis must be achieved. Trochars are placed into the amniotic cavity using a Seldinger approach and a flexible fetoscope introduced into the amniotic cavity $(25,26)$. Balloon tipped trochars can be used to minimize damage to the uterine wall and membranes (27). Following conclusion of the procedure, the uterine access sites and the maternal abdominal trochar sites are closed.

A randomized control trial in Europe known as the Eurofetus trial found that fetoscopic laser photocoagulation in twin-twin transfusion syndrome had superior results when compared to standard therapy of amnioreduction with a higher percentage of infants alive without major neurological morbidity at six months (28). Although no good data exists on its efficacy as compared to open fetal surgery, fetoscopic surgery has been used in treatment of multiple other common fetal abnormalities, including repair of MMC (29). In Europe, fetoscopic endoluminal tracheal occlusion (FETO) is used to treat severe CDH and has been found to have improved survival benefits compared to historical controls receiving standard postnatal therapy,
$55 \%$ versus $8 \%$ (30). Fetoscopic surgery has also been shown to have decreased maternal morbidity as compared to open fetal surgery. In a large retrospective study of 187 women undergoing intrauterine fetal intervention there was significantly less maternal morbidity in terms of length of stay, need for transfusion, and need for ICU stay as compared with open fetal surgery (31). However, there was no difference in the incidence of preterm rupture of membranes or preterm labor (31). Fetoscopic surgery was also shown to have an increased incidence of chorioamniotic separation (31). Fetoscopic surgery shows promise as a less morbid method for intrauterine fetal intervention, but randomized controlled clinical trials (RCTs) are needed to determine its true efficacy, particularly in the treatment of severe CDH.

\section{MMC}

One of the most promising applications of fetal surgery is in the treatment of MMC. MMC is a congenital defect of the central nervous system in which the meninges and spinal cord protrude through a defect in the vertebral arch. This results in paralysis as well as varying degrees of mental retardation, bowel, and bladder dysfunction. Prior to the development of intrauterine therapy, the only available treatment for MMC was closure of the spinal cord at birth. However, the resulting neurological deficits are permanent and patients require lifelong supportive care. Although the exact etiology of MMC is unknown, studies of fetuses suggest that the spinal cord is relatively normal initially and becomes damaged as a secondary result of its exposure to amniotic fluid or some other intrauterine factor (32). In a lamb model, where a MMC type lesion was created early in gestation and then subsequently repaired, there was significantly less neurological deficits in the lambs that underwent in-utero repair (33). This concept was applied to the treatment of human fetuses with MMC, culminating in the landmark Management of Myelomeningocele Study (MOMS) trial. The MOMS trial was a RCT of 183 women with fetuses affected by MMC comparing fetal operative repair with standard post-natal repair (34). Operative repair involved open fetal surgery with closure of the native dura, myofascial flaps, and skin over the defect (34). The trial was terminated early due to the significantly superior outcomes in the fetal intervention group. The study found that infants in the surgical group were $50 \%$ less likely to require ventriculoperitoneal shunt and had a significant decrease in the incidence of the Chiari malformation (34). 
In addition, $42 \%$ of infants were walking independently at 30 months compared to $21 \%$ in the standard therapy group, a significant improvement (34). In long term followup, although children who underwent fetal intervention did have neurodevelopmental delay, the deficits were less severe than those seen in children who had not undergone in-utero repair (35).

Since the MOMS trial, techniques have continued to evolve, including the use of fetoscopic repair rather than open repair and several case reports of fetoscopic repair have been published. A recent case series described 10 fetuses with MMC who underwent completely percutaneous fetoscopic repair with 7 surviving long term. Out of the infants that survived long term, 6 demonstrated complete reversal of hindbrain herniation (36). Although further study is still necessary, fetoscopic repair is less invasive with potentially decreased risk of premature delivery. Fetal intervention in MMC offers the potential to repair a devastating fetal malformation that has frequently been met with poor postnatal operative outcomes.

\section{CLMs}

Congenital fetal lung malformations include congenital cystic adenomatoid malformations (CCAM), bronchopulmonary sequestrations (BPS), and hybrid lesions. These lesions have the capacity for unpredicatble growth during midgestation and must be followed closely with serial US during this growth phase. The majority of these lesions will plateau in their growth and normal lung growth will out pace that of the lung mass in the 3 rd trimester. Smaller lesions will not cause respiratory symptoms at birth, and infants will undergo post-natal resection at 2-6 months of age (26,37). However, larger lesions can create significant mass effect in the chest with cardiomediastinal shift and compression of the normal lung. The most severe lesions can progress to cause fetal hydrops, an almost universal predictor of impending fetal death (26).

Although the majority of fetuses survive to delivery and undergo successful post-natal therapy, fetuses with large lesions and particularly those that develop hydrops will require fetal intervention. Sonographic measurements such as the cystic adenomatoid malformation volume ratio (CVR) are used to risk stratify lesions and identify fetuses who will require in-utero treatment. A CVR of $>1.6$ has an $80 \%$ risk of developing hydrops (22). These high risk microcystic lesions are initially treated with administration of maternal betamethasone to arrest further growth of the lesion. Invasive treatments are reserved for cases of CLMs with associated hydrops. Thoracoamniotic shunts are used to treat lesions with a dominant macrocyst and associated hydrops. These shunts are placed under ultrasound guidance. Microcystic/solid lesions that do not respond to maternal steroid treatment will require open fetal resection (24). A series of 22 fetuses with large, predominantly solid CCAMs who underwent fetal resection had 50\% long term survival with resolution of hydrops in all 11 surviving fetuses (22). Minimally invasive techniques may become possible with further technological advances, including fetoscopic laser fulguration (38).

\section{CDH}

$\mathrm{CDH}$ occurs in 1 in 3,500 live births and the hernia is on the left in almost $90 \%$ of cases (39). Herniation of abdominal contents into the chest impairs normal lung development resulting in pulmonary hypoplasia and pulmonary hypertension (40). With overall CDH survival rates approaching $90 \%$ in high volume centers using standardized post-natal treatment protocols and gentle ventilation strategies, planned delivery and post-natal therapy may be appropriate for the majority of fetuses diagnosed antenatally with $\mathrm{CDH}$. However, there remains a severe CDH subgroup with survival rate less than $25 \%$. Liver position and lung-head ratio (LHR) are important prenatal prognostic measures used to identify severe $\mathrm{CDH}$ fetuses who may benefit from fetal intervention $(41,42)$. A lung-head ratio (LHR) of $<1.2$ has been shown to be an indicator of extremely poor prognosis, with survival of $0-38 \%$ (43). Fetuses with these prognostic factors may benefit from fetal intervention given their likely dismal outcomes with standard post-natal therapy.

FETO has shown early promise in the treatment of severe CDH. A fetoscope is used to deploy a balloon in the fetal trachea. Obstruction of the trachea prevents the egress of lung fluid which has the effect of stimulating lung growth. The balloon is then decompressed by ultrasound guided needle aspiration at approximately 34 weeks gestation. European investigators have shown increased survival in left $\mathrm{CDH}$ from $24 \%$ to $49 \%$ when compared to historical controls from their $\mathrm{CDH}$ registry (25). However, initial RCTs did not show an outcome difference between FETO and standard post-natal treatment (30). The "Tracheal Occlusion to Accelerate Lung Growth" (TOTAL) trial is a multicenter randomized trial in Europe and North America to compare FETO versus standard 
post-natal care. Centers in the United States are in the early phases of enrolling and treating patients at the time of this publication.

\section{Conclusions}

Advances in fetal imaging have markedly improved prenatal diagnostic accuracy and paved the way for the development of novel in-utero interventions. Improving ultrasound technology offers remarkable precision for image guided fetal intervention techniques. As minimally invasive techniques and equipment evolve, the indications for fetal therapy are likely to expand.

\section{Acknowledgements}

None.

\section{Footnote}

Conflicts of Interest: The authors have no conflicts of interest to declare.

\section{References}

1. Canfield MA, Honein MA, Yuskiv N, et al. National estimates and race/ethnic-specific variation of selected birth defects in the United States, 1999-2001. Birth Defects Res A Clin Mol Teratol 2006;76;747-56.

2. Jones KL. Morphogenesis and dysmorphogenesis. In: Smith DW. editor. Recognizable patterns of human malformations. Philadelphia: WB Saunders, 1997:695-705.

3. Souka AP, Pilalis A, Kavalakis I, et al. Screening for major structural abnormalities at the 11 to 14 week ultrasound scan. Am J Obstet Gynecol 2006;194;393-6.

4. Pratt R, Deprest J, Vercauteren T, et al. Computerassisted surgical planning and intraoperative guidance in fetal surgery: a systematic review. Prenat Diagn 2015;35;1159-66.

5. Yagel S, Cohen SM, Shapiro I, et al. 3D and 4D ultrasound in fetal cardiac scanning, a new look at the fetal heart. Ultrasound Obstet Gynecol 2007;29;81-95.

6. Kurjak A, Miskovic B, Andonotopo W, et al. How useful is $3 \mathrm{D}$ and $4 \mathrm{D}$ ultrasound in prenatal medicine? J Perinat Med 2007;35;10-27.

7. Filkins K, Koos B. Ultrasound and fetal diagnosis. Curr Opin Obstet Gynecol 2005;17;185-95.

8. Hirose S, Harrison M. The ex utero intrapartum treatment
(EXIT) procedure. Semin Neonatol 2003;8;207-14.

9. Adzick NS. Prospects for fetal surgery. Early Hum Dev 2013;89;881-6.

10. Dolkart L, Harter M, Snyder M. Four-dimensional ultrasonographic guidance for invasive obstetric procedures. J Ultrasound Med 2005;24;1261-6.

11. Pugash D, Brugger PC, Bettelheim D, et al. Prenatal ultrasound and fetal MRI: the comparative value of each modality in prenatal diagnosis. Eur J Radiol 2008;68;214-26.

12. Coakley FV. Role of magnetic resonance imaging in fetal surgery. Top Magn Reson Imaging 2001;12;39-51.

13. Coakley FV, Hricak H, Filly RA, et al. Complex fetal disorders: effect of MR imaging on managementpreliminary clinical experience. Radiology 1999;213;691-6.

14. Zamora IJ, Sheikh F, Cassady CI, et al. Fetal MRI lung volumes are predictive of perinatal outcomes in fetuses with congenital lung masses. J Pediatr Surg 2014;49;853-8.

15. Werner H, Lopes dos Santos JR, Fontes R, et al. Virtual bronchoscopy for evaluating cervical tumors of the fetus. Ultrasound Obstet Gynecol 2013;41;90-4.

16. Marwan A, Crombleholme T. The EXIT procedure: principles, pitfalls, and progress. Semin Pediatr Surg 2006;15;107-15.

17. Bouchard S, Johnson MP, Flake AW, et al. The EXIT procedure: experience and outcome in 31 cases. J Pediatr Surg 2002; 37;418-26.

18. Hirose S, Farmer DL, Lee H, et al. The ex utero intrapartum treatment procedure: looking back at the EXIT. J Pediatr Surg 2004;39;375-80.

19. Adzick NS, Harrison MR, Flake AW, et al. Automatic uterine stapling devices in fetal operation: experience in a primate model. Surg Forum 1985;36;479-80.

20. Noah MM, Norton ME, Sandberg P, et al. Short-term maternal outcomes that are associated with the EXIT procedure, as compared with cesarean delivery. Am J Obstet Gynecol 2002;186;773-7.

21. Zamora IJ, Ethun CG, Evans LM, et al. Maternal morbidity and reproductive outcomes related to fetal surgery. J Pediatr Surg 2013;48;951-5.

22. Adzick NS. Open fetal surgery for life-threatening fetal anomalies. Semin Fetal Neonatal Med 2010;15;1-8.

23. Lovvorn HN, Crombleholme TM, Drott H, et al. Maternal colloid osmotic pressure after fetal surgery. Surg Forum 1998;49;481-3.

24. Wilson RD, Johnson MP, Crombeholme TM, et al. Chorioamniotic membrane separation following open fetal surgery: pregnancy outcomes. Fetal Diagn Ther 2003;18;314-20. 
25. Deprest J, Jani J, Lewi L, et al. Fetoscopic surgery: Encouraged by clinical experience and boosted by instrument innovation. Semin Fetal Neonatal Med 2006;11;398-412.

26. Adzick NS, Harrison MR, Crombleholme TM, et al. Fetal lung lesions: management and outcome. Am J Obstet Gynecol 1998;179;884-9.

27. Kohl T, Hering R, Van de Vondel P, et al. Analysis of the stepwise clinical introduction of experimental percutaneous fetoscopic surgical techniques for upcoming minimally invasive fetal cardiac interventions. Surg Endosc 2006;20;1134.

28. Senat MV, Deprest J, Boulvain M, et al. Endoscopic laser surgery versus serial amnioreduction for severe twin-to-twin transfusion syndrome. N Engl J Med 2004;351;136-44.

29. Belfort MA, Whitehead WE, Shamshirsaz AA, et al. Fetoscopic repair of meningomyelocele. Obstet Gynecol 2015;126;881-4.

30. Harrison MR, Keller RL, Hawgood SB, et al. A randomized trial of fetal endoscopic tracheal occlusion for severe fetal congenital diaphragmatic hernia. N Engl J Med 2003;349;1916-24.

31. Golombeck K, Ball RH, Lee H, et al. Maternal morbidity after maternal-fetal surgery. Am J Obstet Gynecol 2006;194;834-9.

32. Korenromp MJ, Van Gool JD, Bruinese HW, et al. Early fetal leg movements in myelomeningocele. Lancet 1986;1;917-8.

33. Meuli M, Meuli-Simmen C, Hutchins GM, et al. In utero repair of experimental myelomeningocele saves neurological function at birth, J Pediatr Surg 1996;31;397-402.

34. Adzick NS, Thom EA, Spong CY, et al. A randomized trial

Cite this article as: Maselli KM, Badillo A. Advances in fetal surgery. Ann Transl Med 2016;4(20):394. doi: 10.21037/ atm.2016.10.34 of prenatal versus postnatal repair of myelomeningocele. N Engl J Med 2011;364;993-1004.

35. Johnson MP, Gerdes M, Rintoul N, et al. Maternal-Fetal surgery for myelomeningocele: neurodevelopmental outcomes at 2 years of age. Am J Obstet Gynecol 2006;194;1145-50.

36. Pedreira DA, Zanon N, Nishikuni K, et al. Endoscopic surgery for the antenatal treatment of myelomeningocele: the CECAM trial. Am J Obstet Gynecol 2016;214;111.e1-11.

37. MacGillivray TE, Harrison MR, Goldstein RB, et al. Disappearing fetal lung lesions. J Pediatr Surg 1993;28;1321-4.

38. Milner R, Kitano Y, Olutoye O, et al. Radiofrequency thermal ablation: a potential treatment for hydropic fetuses with a large chest mass. J Pediatr Surg 2000;35;386-9.

39. Langham MR, Kays DW, Ledbetter, et al. Congenital diaphragmatic hernia: epidemiology and outcome. Clin Perinatol 1996;23;671-88.

40. Metkus AP, Filly RA, Stringer MD, et al. Sonographic predictor of survival in fetal diaphragmatic hernia. J Pediatr Surg 1996;31;148-51.

41. Albanese CT, Lopoo J, Goldstein RB, et al. Fetal liver position and perinatal outcome for congenital diaphragmatic hernia. Prenat Diagn 1998;18;1138-42.

42. Metkus AP, Esserman L, Sola A, et al. Cost per anomaly: what does a diaphragmatic hernia cost. J Pediatr Surg 1995;30;226-30.

43. Lipshutz GS, Albanese CT, Feldstein VA, et al. Prospective analysis of lung-to-head ratio predicts survival for patients with prenatally diagnosed congenial diaphragmatic hernia. J Pediatr Surg 1997;32;1634-6. 